\title{
A importância da lavanderia no controle da infecção em um hospital de pequeno porte
}

\section{Carlos da Silva Marques}

Graduado em Administração Hospitalar pela Faculdade de Ciências Sociais e Humanas de Garanhuns. Email: carlinhosww@msn.com

\section{Cleide Calado de Araújo.}

Especialista em Enfermagem. Professora da Autarquia de Ensino Superior de Arcoverde - AESA.

Email: cleideeu@hotmail.com

\section{Elisângela de Sousa Branco.}

Doutora em Oceanografia Biológica pela Universidade Federal de Pernambuco - UFPE (2007) e mestre em Oceanografia Biológica pela Universidade Federal de Pernambuco. Professora efetiva da Autarquia de Ensino Superior de Arcoverde e da Autarquia de Ensino Superior de Garanhuns (2009).

Email: brancoesb@hotmail.com

RESUMO; Dentre a estrutura organizacional de uma instituição de saúde, a lavanderia hospitalar é um dos principais serviços de apoio ao atendimento dos pacientes, na qual é responsável pelo processamento da roupa e sua distribuição em perfeitas condições de higiene e conservação. Um bom sistema de processamento da roupa é um fator de redução das infecções hospitalares e impactos ambientais. É de grande importância o estudo dos fatores que propiciam e acometem os pacientes de uma unidade hospitalar perante as infecções adquiridas neste meio, para que se possam obter indicadores para possíveis controles. Este estudo foi realizado através de levantamento bibliográfico de artigos científicos. Trata da determinação de diretrizes e parâmetros para orientar a contratação dos Serviços de Lavanderia Hospitalar, para processamento de roupas e tecidos em geral em todas as suas etapas, desde sua utilização até seu retorno em condições de reutilização, sob situações higiênico-sanitárias adequadas, mediante a operacionalização e execução de todas as atividades. A legislação pertinente à lavanderia está condicionada ao Manual de Lavanderia Hospitalar do Ministério da Saúde (1986) e suas atualizações, que é um trabalho com uma série de instrumentos normativos elaborados com a finalidade de atender requisitos tais como: controle das infecções; recuperação, conforto e segurança do paciente; facilidade, segurança e conforto da equipe de trabalho; racionalização de tempo e material; redução dos custos operacionais. Esse manual engloba as rotinas e as técnicas, ou métodos administrativos empregados na realização deste trabalho.

PALAVRAS-CHAVES: Lavanderia Hospitalar, Infecção Hospitalar, Processamento de Roupas.

\section{The importance of laundry in the control of infection in small hospital}

ABSTRACT: Among the organizational structure of a health institution, the hospital laundry is a major support services for patient care, which is responsible for processing and distribution of clothes in perfect conditions of hygiene and conservation. A good system of processing of clothing is a factor to reduce nosocomial infections and environmental impacts. It is of great importance to study the factors that promote and affect patients in a hospital before the infections acquired in this way, so you can get indicators of possible controls. This study was conducted through literature review of scientific articles available on the Internet. Deals with the determination of guidelines and parameters to guide the recruitment of Hospital Laundry Services, for processing of clothing and textiles in general in all its stages, from its use until his return in perfect condition for reuse under appropriate hygienic and sanitary conditions, through the operationalization and implementation of all activities. The relevant legislation is subject to the laundry to the Manual of Hospital Laundry Ministry of Health - 1986 and its updates, which is working with a series of normative instruments developed in order to meet the following requirements such as: infection control; recovery comfort and patient safety, ease, safety and comfort of the work team; rationalization of time and material, reducing operating costs. This manual covers the routines and techniques, or administrative methods employed in this work.

KEY WORDS: Hospital Laundry, Hospital Infection, processing clothing 


\section{INTRODUÇÃO}

Antes de conceituar a lavanderia é necessário que se defina o que é hospital de pequeno porte. Uma unidade hospitalar caracteriza-se como sendo de pequeno porte, quando contém menos de 50 (cinquenta) leitos e for composta pela seguinte estrutura organizacional: diretoria administraiva; clínica médica; clínica odontológica; clínica radiológica; clínica de recuperação física ou mental; ambulatório; pronto-socorro; postos de saúde ou puericultura; bancos de sangue ou laboratórios de análise. (ANVISA, 2007)

Como a maioria das empresas, a unidade hospitalar é dividida em vários setores, que são essenciais para o bom funcionamento da instituição, dentre esta estrutura organizacional destacamos o setor lavanderia por sua importância no controle das infecções como também na oferta de roupas de cama limpas e confortáveis para os clientes internos e externos, proporcionando além da redução no índice de infecções, a melhora no atendimento ao hospedar o usuário deste serviço.

A lavanderia hospitalar é um dos principais serviços de apoio ao atendimento dos pacientes, responsável pelo processamento da roupa e sua distribuição em perfeitas condições de higiene e conservação. Um bom sistema de processamento da roupa é fator de redução das infecções hospitalares e impactos ambientais. (MACÊDO et al., 2002)

Segundo Mesiano e Lisboa (2006), as atividades existentes em lavanderias hospitalares são: coleta e armazenamento, transporte, pesagem, separação, classificação, lavagem, centrifugação, calandragem, secagem, prensagem e de rouparia. E ainda mencionam que cada atividade apresenta características e necessidades físicas, tecnológicas e humanas para o seu desenvolvimento.

O controle e uso adequado da temperatura, umidade, luminosidade, insolação, ventos dominantes e renovação de ar contribuem para o conforto dos servidores. A lavanderia é uma área que compreende um conjunto de maquinário característico que geralmente provoca muito ruído e vibração, devendo, portanto, ter tratamento acústico enquanto que as máquinas devem ser fixadas ao piso a fim de diminuir a transmissão das vibrações. (BARTOLOMEU, 1998)

De acordo com Castro e Chequer (2001), toda roupa hospitalar, independente do grau de sujidade, é considerada contaminada, em razão do próprio ambiente e da presença de pacientes portadores ou não de doenças infectocontagiosas e do seu acompanhante. Assim, todo o processo de lavagem da roupa hospitalar deve conter uma etapa de desinfecção, seja térmica ou química.

A lavanderia hospitalar tem como objetivo transformar toda a roupa suja ou contaminada, utilizada no hospital, em roupa limpa. Este processo é extremamente importante para o bom funcionamento do hospital em relação à assistência direta ou indireta prestada ao paciente. E para Arsego et al (2008) o processamento de roupas dentro dos hospitais deve ser dirigido de forma que a roupa não represente um veículo de infecção, contaminação ou mesmo irritação aos pacientes e funcionários.

Portanto, este trabalho tem como objetivo geral analisar os fatores que interferem no funcionamento adequado da lavanderia hospitalar, tendo como objetivo específico indicar a importância da mesma na diminuição dos riscos de infecções hospitalares.

\section{A ADMINISTRAÇÃO DA LAVANDERIA HOSPITALAR}

É de fundamental importância a administração da lavanderia hospitalar, pois, de acordo com a ANVISA (2007), o objetivo da administração da lavanderia hospitalar é contribuir para a segurança e bem-estar do cliente interno e externo, e para a otimização do padrão do hospital. A administração compreende as seguintes fases de planejamento: organização, coordenação, direção ou comando, controle e administração de material.

Na organização de uma lavanderia hospitalar, todo o seu funcionamento deve estar descrito em um manual de orientação, manual este que está disponível no site do Ministério da Saúde. Este manual deve conter a especificação de cada atividade, a estrutura hierárquica, regras e usualidades, expondo nitidamente a organização e execução das tarefas. As funções desempenhadas pela equipe que compõe a lavanderia devem constar no regulamento do hospital. A mesma lavanderia deve estar diretamente subordinada à administração da unidade hospitalar, fazendo parte dos serviços gerais.

A área de recebimento, separação, pesagem e lavagem das roupas sujas na lavanderia são consideradas uma das mais contaminadas de todo o hospital. A área caracteriza-se por apresentar odor, risco de contaminação e fadiga. (BARTOLOMEU, 1998) 
Os equipamentos usados em uma lavanderia hospitalar normalmente são: lavadora de desinfecção, centrífuga ou extratora, lavadora extratora, calandra, secadora, prensa, balança, carro de transporte e máquina de costura. Esse maquinário geralmente provoca muito ruído, vibração e calor.

E ainda para Konkewicz (2006) o processamento das roupas hospitalares abrange todas as etapas pelas quais as roupas passam, desde sua utilização até seu retorno em ideais condições de reuso. Estas etapas são geralmente classificadas em seleção, acondicionamento, coleta e transporte da roupa suja utilizada nos diferentes setores do hospital; recebimento e lavagem da roupa suja na lavanderia; secagem e calandragem da roupa limpa; separação e transporte da roupa limpa da lavanderia para os diversos setores do hospital; armazenamento e controle de estoque da roupa limpa nos setores do hospital. Também podem estar incluídas neste processo a confecção e o reparo das roupas.

Já para Borba (2006) na organização interna das divisões, dos departamentos e serviços, o hospital deve embasar-se em instrumentos normativos próprios, comumente chamados de regimentos, ou manual de normas do departamento. Este instrumento deve ser elaborado em consonância com os dispositivos regulamentados no instrumento superior e que the proporciona formalidade organizacional pelo Ministério da Saúde.

Conforme as normas do Ministério da Saúde (1986), o organograma do serviço de lavanderia reflete a organização, comando, subordinação e distribuição de atividades, como segue abaixo, dentro dos seguintes setores:

- Área suja: coleta, separação ou triagem, pesagem e lavagem.

- Área limpa - setor de acabamento: centrifugação, secagem, calandragem e prensagem.

- Rouparia: costura, estocagem e distribuição.

\subsection{Coordenação}

A coordenação única da lavanderia permite a obtenção de um funcionamento harmonioso e de uma produção eficiente.

\subsection{Direção ou Comando}

Nesta etapa da administração, os recursos são gerenciados e dinamizados visando o eficaz funcionamento do serviço de lavanderia. A direção e coordenação são, em geral, exercidas pelo mesmo indivíduo.

Essas funções devem funcionar de forma interdisciplinar sob uma única chefia administrativa, com plena autoridade em todas as fases do processamento da roupa, desde a coleta até a sua redistribuição. A descrição das funções e respectivas tarefas definem os deveres de cada profissional, permitindo a racionalização do trabalho.

\subsection{Controle}

O eficaz controle administrativo acarreta o uso completo dos recursos para a conquista dos objetivos e metas pré-estabelecidas, a custos operacionais adequados. Os mecanismos de controle mais usados na lavanderia são a supervisão e a avaliação.

- Supervisão - a supervisão na lavanderia hospitalar, realizada em caráter de constante observação e orientação do pessoal, contribui para o desenvolvimento da equipe de trabalho e a racional eficácia do serviço;

- Avaliação - mede a adaptação e eficiência do serviço, comprova o alcance dos objetivos e metas, e orienta o emprego de medidas para a correção de desvios.

\subsection{Administração de Pessoal}

A administração de pessoal tem como objetivo analisar diferentes modalidades de gestão institucional que combinem liberdade e autonomia dos trabalhadores da saúde, em especial a dos profissionais, com controle institucional e atribuição de responsabilidade. Tendo como funções administrativas, de acordo com Nascimento (2009), relacionadas ao pessoal:

- Recrutamento e Seleção - é importante considerar o estado saudável, a capacitação e/ou experiência anterior do candidato, a preferência pelo tipo de trabalho, as condições de $\mathrm{RH}$ e a nível profissional compatível com as funções a serem desempenhadas. Apesar de existirem diversos meios de avaliar um profissional que está concorrendo a uma oportunidade, a entrevista continua sendo a principal técnica utilizada, e, muitas vezes, a decisiva. É através deste método que se pode perceber e conhecer mais profundamente o entrevistado 
- Treinamento - para o bom desempenho na lavanderia, o servidor integrado a equipe deve receber um treinamento inicial, mesmo que disponha de experiência na área ou possua curso específico. A finalidade do treinamento é capacitar cada membro da equipe para a execução eficiente de suas tarefas.

Observação: em caso da lavanderia que sirva a outros estabelecimentos da rede, o chefe ou encarregado deve também treinar o pessoal de coleta e transporte da roupa.

- Direção de Pessoal - é necessário que o chefe tenha espírito de liderança, bom relacionamento com a equipe, sempre buscando a eficiência do pessoal.

- Controle - é uma das mais importantes e difíceis funções da administração de pessoal, a qual exige habilidade, percepção e sensibilidade por parte de quem a realiza. É uma função crítica do processo administrativo. Os instrumentos mais frequentemente utilizados no controle de pessoal são:

- Supervisão: deve funcionar como um sistema educativo e de ajuda ao crescimento do servidor. O resultado da supervisão deve ser registrado em fichas individuais, para cada servidor.

- Avaliação: existem vários dispositivos para avaliar o desempenho do servidor. Dentre estes, há o registro em "cartão de produção", após cada hora ou jornada de trabalho, que permite a comparação com a produtividade dos demais servidores ou com a dos "tempos-padrões".

- Previsão de Pessoal - o número de membro da equipe ou o quadro de pessoal da lavanderia dependem dos equipamentos, instalações e métodos de desempenho do serviço. Pode haver racionalização de mão-de-obra através da instalação de equipamento mais produtivo, de suprimento de água, vapor e energia elétrica em qualidade e quantidade necessárias e adequado abastecimento de material em geral.

\subsection{Administração de Material}

Os materiais constituem, em geral, segundo Beulke e Bertô (2006), elemento relevante no custo. A gestão de materiais pode ocorrer no aspecto físico e monetário. Monetariamente, isso ocorre pela pesquisa, busca e cotação dos materiais mais favoráveis do ponto de vista de custos. No aspecto físico o melhor controle deve ser efetuado sobre os níveis de estocagem e sobre os níveis de perdas.

O material representa o apoio logístico necessário ao bom funcionamento do serviço. A administração de material compreende uma série de funções administrativas, destacando-se, na lavanderia, as seguintes:

- Previsão - ao se prever o material para a lavanderia, deve-se fazer um plano do que é necessário e um estudo do que é oferecido pelos fornecedores, para se decidir pelo mais adequado, conveniente e menos oneroso. Recomenda-se a padronização dos materiais, principalmente da roupa e dos produtos. É importante ressaltar que existe uma correlação entre o material e os procedimentos adotados. A previsão do material deve ser realizada sistematicamente, em períodos regulares (trimestrais, semestrais ou anuais), para assegurar o abastecimento da lavanderia;

- Provisão - os materiais necessários à lavanderia devem ser solicitados ao almoxarifado em períodos preestabelecidos, mediante requisições em formulários próprios;

- Controle - o material pode ser controlado por meio de fichas de entrada e saída, boletins estatísticos de gastos e de produtividade, supervisão, auditoria e levantamentos periódicos. A marcação da roupa com a identificação do hospital é também um método de controle muito utilizado, oferecendo informações e evitando o desvio de peças.

\section{FATORES QUE INFLUENCIAM NO AUMENTO DAS INFECÇÕES}

As roupas em unidades hospitalares representam todo e qualquer material de tecido utilizado no âmbito hospitalar e que necessitam passar por um rigoroso processo de lavagem e secagem para sua reutilização. Roupas hospitalares, por exemplo, incluem lençóis, fronhas, cobertores, toalhas, colchas, cortinas, roupas de pacientes e roupas de funcionários, fraldas em tecido, compressas, campos cirúrgicos, máscaras, aventais, gorros, panos de limpeza, entre outros. Com esses exemplos pode-se perceber a grande diversidade, procedência, diferentes empregos, sujeiras e contaminação das roupas utilizadas dentro de hospitais.

De acordo com Cargnin (2008), as roupas hospitalares diferem daquelas utilizadas em outros tipos de instituições ou residências porque alguns itens apresentam-se contaminados com materiais biológicos de pacientes em maior quantidade de contaminação e volume de roupa, mas não há distinção das sujeiras encontradas nas roupas da comunidade em geral. 
O processamento das roupas hospitalares abrange todas as etapas pelas quais as roupas passam, desde sua utilização até seu regresso em ideais condições de reutilização.

Segundo Macêdo et al (2002) estas etapas são geralmente classificadas em: coleta (no expurgo) e transporte da roupa contaminada utilizada nos distintos setores do hospital; recebimento e lavagem da roupa suja na lavanderia; secagem e calandragem da roupa limpa; separação e transporte da roupa limpa da lavanderia para a rouparia do hospital. Também podem estar incluídas neste processo a locação e o reparo das roupas.

A contaminação da roupa hospitalar conforme Konkewicz (2006) depende basicamente da quantidade de sua sujidade e da proveniência desta sujidade. Roupas sujas de fezes, secreções purulentas, urina, sangue, secreções vaginais, uretrais, gástricas e outras secreções e excreções corporais apresentarão muito maior quantidade de microrganismos do que roupas com sujidade não proveniente de pacientes, como alimentos, líquidos diversos, poeira, etc. Quanto maior a quantidade da sujidade, também obviamente maior será a quantidade de microrganismos presentes na roupa suja.

As roupas hospitalares diferem daquelas utilizadas em outros tipos de instituições ou residências porque alguns itens apresentam-se contaminados com sangue, secreções ou excreções de pacientes em maior quantidade de contaminação e volume de roupa, mas não diferentemente das sujidades encontradas nas roupas da comunidade em geral.

Mesmo que o processo de lavagem, centrifugação, secagem e calandragem da roupa sejam os mais adequados possíveis, o resultado final não representa eliminação total de microrganismos, já que não significa um processo de esterilização. (BARRIE, 1994)

Conforme o mesmo autor a meta principal a ser atingida após o processamento da roupa deve ser a redução das contagens microbianas para níveis aceitáveis, ou seja, livre de patógenos em quantidade e qualidade suficientes para transmitir doenças.

Apesar da roupa suja ser identificada como fonte de um largo número de microrganismos patogênicos, o risco de transmissão de doenças para os trabalhadores do hospital e para os pacientes que mantém contato direto com a roupa é negligenciável (STANDAERT; HUTCHESON; SCHAFFNER, 1994). Poucos estudos, e não muito recentes, relatam infecção cruzada associada com roupa em pacientes e em trabalhadores de hospitais.

Conforme Santos e Messiano (2008), o contato direto com as secreções e fluidos corporais presentes nas roupas sujas e a aspiração do ar contaminado por aerossóis gerados pela agitação das peças constituem os principais mecanismos de transmissão de doenças infecciosas durante o processamento destas roupas.

As mesmas autoras complementam dizendo que a contaminação dos funcionários e do ambiente tem maior probabilidade de ocorrer nas seguintes situações: no momento em que as peças são retiradas de seus locais de uso; durante o transporte, quando o ensacamento não for realizado corretamente; se houver cruzamento real de roupas sujas e limpas; e quando a utilização dos Equipamentos de Proteção Individuais for negligenciada.

A contaminação pode ser evitada pelo manuseio cuidadoso das peças, com a adoção das precauções para fluidos e secreções corporais aplicadas a todas as roupas, independente da origem, e com o processamento das roupas obedecendo as rotinas técnicas, seguindo as normas do Ministério da SDesde que adequadamente manuseadas e processadas, as roupas não constituem um risco aumentado na transmissão de doenças.

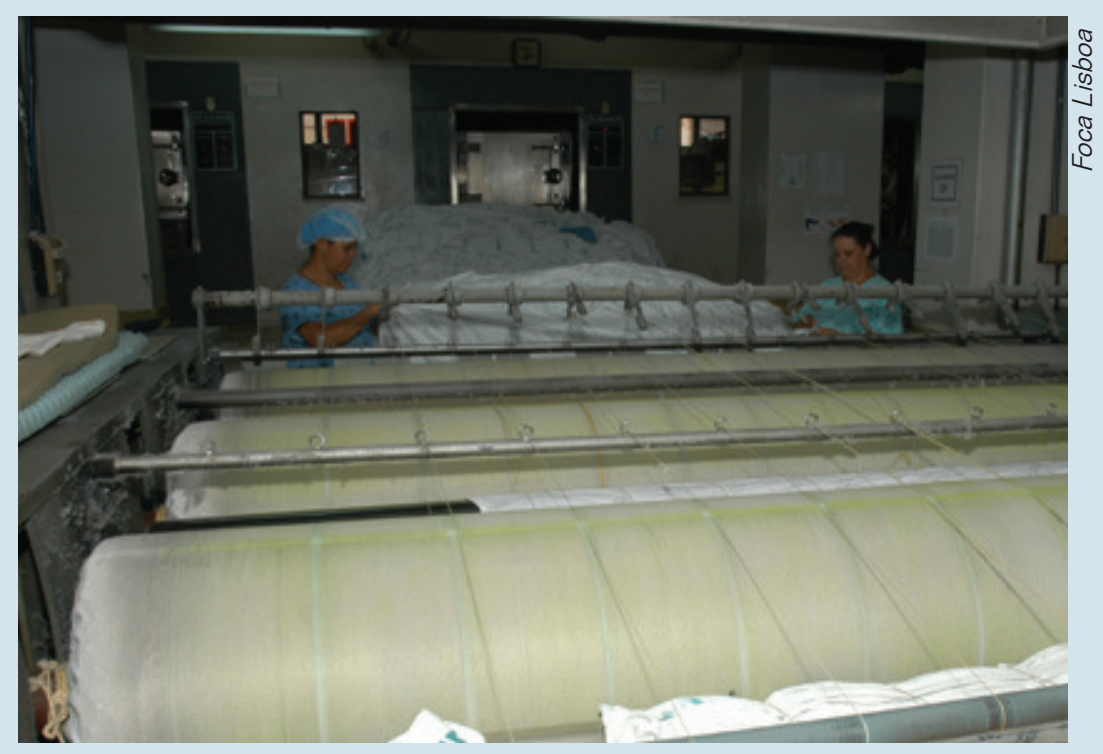




\section{ESTERILIZAÇÃO DAS ROUPAS HOSPITALARES}

Quando um paciente é recebido em um leito limpo, às vezes é difícil imaginar que as roupas que estão sendo utilizadas já serviram a outros. Quando um campo cirúrgico é aberto, impecavelmente estéril, nem sempre lembramos que, horas antes, ele provavelmente estava coberto de sangue, secreções e líquidos diversos.

Mas a sensação de bem estar e confiança, ao se utilizar as roupas hospitalares, será o resultado do bom desempenho da lavanderia no cumprimento de seu principal objetivo: transformar a roupa suja e contaminada em roupa limpa, na quantidade necessária, em um tempo adequado e com segurança. As roupas não precisam estar estéreis ao final deste processo, mas necessitam estar higienicamente limpas, livres da quantidade de microrganismos patogênicos necessária para causar doença.

Como já foi citado anteriormente, o processamento normal da roupa não resulta em eliminação total dos microrganismos, especialmente em suas formas esporuladas. Para isto, seria necessário um processo de esterilização, preferencialmente através de autoclavação a vapor e pressão. Quando existe possibilidade da roupa entrar em contato com pele não íntegra, áreas cruentas, mucosas e tecidos expostos, necessariamente devem ser prevista sua esterilização. Isto se aplica para procedimentos cirúrgicos, procedimentos invasivos, em queimaduras e outras situações em que ocorra a quebra da barreira de proteção da pele. (KONKEWICZ, 2006)

Mesmo em se tratando de pacientes imunodeprimidos, a necessidade de esterilização das roupas se aplica somente para as situações relatadas anteriormente. Esta é uma prática que vem sendo seguida por muitos hospitais brasileiros.

\section{CONSIDERAÇÕES FINAIS}

A infecção hospitalar apresenta-se como um agravo de grande significado epidemiológico, dentro do contexto da assistência hospitalar. Suas consequências são relevantes, querem do ponto de vista humano, querem do ponto de vista econômico.

A atenção dos profissionais da saúde deve direcionar-se às medidas profiláticas e de controle da infecção hospitalar, tendo como meta garantir a qualidade da assistência oferecida à comunidade. Para tanto, impõem-se esforços contínuos na busca de soluções eficazes e eficientes.

A adoção de uma filosofia que priorize a prevenção toma-se condição indispensável ao desenvolvimento dos avanços alcançados pela tecnologia, beneficiando o homem em suas duas dimensões: enquanto usuário do hospital e como trabalhador pertencente à equipe de saúde.

Desta forma, acredita-se que este trabalho é de suma importância para refletir a respeito dos fatores que interferem no funcionamento adequado da lavanderia hospitalar, contribuindo assim para diminuição dos riscos das infecções hospitalares.

Em se tratando dos fatores que interferem no funcionamento adequado da lavanderia hospitalar foi observado que quanto maior a quantidade da sujidade, maior será a quantidade de microrganismos presentes na roupa suja, por isto o resultado final não representa eliminação total de microrganismos. Porém, a contaminação pode ser evitada pelo manuseio cuidadoso das peças, na qual as roupas não constituem um risco aumentado na transmissão de doenças. 


\section{REFERÊNCIAS}

ANVISA. Agência Nacional de Vigilância Sanitária. Processamento de roupas de serviços de saúde: prevenção e controle de riscos. Brasília, 2007. 120 p.

ARSEGO, J; POLETTO, AR; MEDEIROS, E; GONTIJO, LA. Riscos Ocupacionais na área contaminada de uma lavanderia Hospitalar. XXVIII Encontro Nacional de Engenharia de Produção. Rio de Janeiro, RJ, Brasil, 13 a 16 de outubro de 2008.

BARRIE D. How hospital linen and laundry services are provided. J Hosp Infection. 1994, 27: 219-235.

BARTOLOMEU, TA. Identificação e avaliação dos principais fatores que determinam a qualidade de uma lavanderia hospitalar. Dissertação. Universidade Federal de Santa Catarina, 1998.

BEULKE, R; BERTÓ, SJ. Gestão de custos. São Paulo: Saraiva, 2006.

BORBA, VR. Teoria geral da administração hospitalar: estruturação e evolução do processo de gestão hospitalar. Rio de Janeiro, 2006.

CARGNIN, MT. Análise do processo de lavagem e desinfecção de roupas hospitalares: o caso da lavanderia do HUSM. Dissertação. Universidade Federal de Santa Maria Centro de Tecnologia. Santa Maria - RS. Brasil. 2008.

CASTRO, RMS; CHEQUER, SSI. Serviço de processamento da roupa hospitalar: gestão e funcionamento. Viçosa: UFV, 2001.

KONKEWICZ, LR. Prevenção e Controle de Infecções Relacionado ao Processamento das Roupas Hospitalares. 16p. 2006.

MACÊDO, RMPR; SANTOS, EM; ROCHA, SS; MELO, MAF, MARQUES-JUNIOR, S. Avaliação de impacto ambiental: um estudo de caso na lavanderia do hospital universitário Ana Bezerra - Santa Cruz - RN. XXII Encontro Nacional de Engenharia de Produção. Curitiba - PR, 23 a 25 de outubro de 2002.

MESIANO, RA; LISBOA, TC. Processamento de roupas em serviços de saúde. 2006. Disponível em: $<$ http://anvisa.gov.br> Acesso em: 16 de abril de 2010.

MINISTÉRIO DA SAÚDE. Manual da lavanderia hospitalar. Brasília: Centro de Documentação do Ministério da Saúde, (Serie A: Normas e Manuais Técnicos). 1986.

NASCIMENTO, RFL. A entrevista de Seleção. 2009. Disponível em: http://www.rh.com.br/Portal/ Recrutamento_Selecao/Artigo/6071/a-entrevista-de-selecao.html acessado em 27 de maio de 2010.

SANTOS, AAM; MESSIANO, ERAB; A Lavanderia e o Controle de Infecção Hospitalar - Epidemiologia e Controle - 1a Ed. - Capitulo 10 - Lavanderia Hospitalar e o controle de Infecções. 2008.

STANDAERT SM, HUTCHESON RH, SCHAFFNER W. Nosocomial transmission of Salmonella gastroenteritis to laundry workers in a nursing home. Infect Control Hosp Epidemiol. 1994, 15: 22-6. 